\title{
Transnasal nimodipine-loaded mucoadhesive nanoliposomes: Preparation and in-vitro evaluation
}

\begin{tabular}{cc}
\hline Nozad Rashid Hussein ${ }^{1 *}$ & Huner Kamal Omer $^{1}$ \\
\hline Abstract &
\end{tabular}

Background and objective: Nimodipineis effective in the treatment of various cerebrovascular impairment, but its clinical potential is limited due to several undesirable characteristics such as low bioavailability which caused by first pass effect in the liver and low aqueous solubility. The main purpose of this study was to prepare nimodipine-loaded nanoliposomes for intranasal delivery and performing in-vitro studies.

Methods: The nimodipine loaded nanoliposome formula was prepared by ethanol-based proliposome method and characterized in term of particle size and size distribution, zeta potential, entrapment efficiency, and permeability studies. In addition, mucoadhesive nanoliposomes loaded nimodipine was prepared using chitosan as a mucoadhesive agent.

Results: The in-vitro studies explored particle size increased (122.48nm \pm 0.002$)$ for chitosan coated formulation after sonication in comparison to non-coated formulations (114.09 $\mathrm{nm} \pm$ 0.025). Also, the zeta potential was positive for the sonicated chitosan-coated formulation $(5.286 \mathrm{mV} \pm 0.341)$ while for non-coated formulations with chitosan were found to be negative $(-1.317 \mathrm{mV} \pm 0.153)$. The entrapment efficiency $(76.033 \% \pm 0.094 \%)$ and drug permeability $(2.53 \mu \mathrm{g} / \mathrm{ml}$ in the duration of $240 \mathrm{~min})$ were significant for chitosan-coated liposome compared to other formulations.

Conclusion: This study concludes that chitosan glutamate (PROTASAN ${ }^{\circledR}$ UPG213) coatednimodipine-loaded liposomes can be considered as a promising novel formulation for an efficient intranasal delivery of nimodipine. Additionally, changes in the size of liposomes and zeta potential confirmed the existence of a coating layer on the surface of liposome pellets. Chitosan was found to significantly enhance the drug entrapment and could also be considered as a permeability enhancer.

Keywords: Mucoadhesive; Nasal; Nimodipine; Nanoliposome.

\section{Introduction}

Nasal drug delivery will eventually grow and emerge as a potential alternative to various routes of administration of numerous drugs. The use of nasal route is highly advantageous for the delivery of drugs in the treatment of local and systemic diseases. ${ }^{1}$ It is a non-invasive route which is considered as an alternative, especially for the systemic drugs which have problems such as low bioavailability or enzymatic degradation when administered through the oral route. ${ }^{2}$ The administered drugs through nasal route have the ability to reach the central nervous system and bypassing the blood brain barrier., ${ }^{1,3}$ Using suitable pharmaceutical carriers such as micelles, nanoparticles, microspheres, or liposomes are necessary to achieve good bioavailability. Among them, liposomes have shown high potential for intranasal administration of drugs. ${ }^{3-5}$ Liposomes are spherical vesicles and have the tendency of entrapping peptide, protein, and various drug molecules. The limitation of liposome is easily removed by mucociliary clearance after nasal delivery. ${ }^{4}$ The mucociliary clearance can be however slowed by the use of polysaccharides which have the ability to prolong the contact time between

${ }^{1}$ Department of Pharmaceutics, College of Pharmacy, Hawler Medical University, Erbil, Iraq.

* Correspondence: nozad.hussein@hmu.edu.krd 
drug and nasal mucosa, thus promoting drug absorption. ${ }^{6,7}$ Among the various polysaccharides, chitosan, and its derivatives are widely used as a mucoadhesive agent, ${ }^{8}$ due to their capability of providing strong mucoadhesion via binding with sialic acidic residues of mucin molecules. ${ }^{9,10}$ Upadhyay et al. developed a formulation of Quetiapine Fumarate loaded nanoliposomes for better diffusion and administration through nasal route to the brain. In vivo studies were carried out in mice demonstrated intranasally administered Quetiapine Fumarate loaded nanoliposomes resulted in higher brain level of the drug compared to simple dispersion. $^{3}$ Wang and coworker in 2006 showed nimodipine loaded liposome produces higher and more stable plasma and cerebral drug concentrations compared with nimodipine solution after intravenous administration. ${ }^{11}$ Nimodipine is a 1, 4-dihydropyridine calcium antagonist which mainly dilates the cerebral arterioles and thus increases cerebral blood flow in animals and humans. ${ }^{11,12}$ It has high potential role in the treatment of various cerebrovascular disorders such as migraine, cerebrovascular spasm, and stroke. ${ }^{13}$ Clinical studies of Nimodipine has shown low oral bioavailability of about $4-13 \%$ in healthy subjects due to the poor aqueous solubility of the drug $(3.86 \mu \mathrm{g} / \mathrm{ml})$ and high first-pass effect in liver. ${ }^{14,15}$ This study aimed to design a novel formulation of nimodipine loaded nanoliposomes using ethanol-based proliposome method. Parameters involved in the preparation were investigated to optimize the loading efficiency of nimodipine in nanoliposomes using two different concentrations of lipid and to establish an optimum formulation for nasal administration. Furthermore, the investigations were carried out for another batch, which included the mucoadhesive polymer(chitosan glutamate) to study its influence on the liposome formulation.

\section{Methods}

\section{Materials}

Nimodipine was purchased from Apollo healthcare, Singapore. Soya phosphatidylcholine (SPC) was obtained from Lipoid, Switzerland. Cholesterol (analytical grade) was purchased from Sigma-Aldrich, UK. All HPLC grade solvents (i.e., water, methanol, 96\% ethanol and acetonitrile) were purchased from Fisher Scientific Ltd., UK. Chitosan glutamate (PROTASAN ${ }^{\circledR}$ UPG213) was obtained from Novamatrix, FMC BioPolymers, Norway. Ultra-thin silicone membrane was purchased from J-flex, UK.

\section{Methods}

\section{Preparation of nimodipine-loaded liposomes}

The method of ethanol-based proliposome was modified from that of Elhissi et al. (2006)by using $4: 1 \mathrm{w} / \mathrm{w}$ of lipid to cholesterol ratio, and increasing the amount of ethanol to lipidratio in order to enhance the dissolution of lipid. Liposomes containing nimodipine were prepared by incorporating two different lipid concentrations (low lipid and high lipid concentration). For low lipid formulation, pellets of liposome were prepared by adding $228 \mu \mathrm{l}$ absolute ethanol into a $15 \mathrm{ml}$ glass vial containing $120 \mathrm{mg}$ lipid of Soya phosphaditylcholine (SPC) at $70^{\circ} \mathrm{C}$ to enhance dissolution of cholesterol (Table 1), SPC and cholesterol $(4: 1 \mathrm{w} / \mathrm{w})$ were used in both low and high lipid formulation. Cholesterol was added to increase vesicle membrane rigidity and to prevent drug leakage. While for high lipid formulation, $240 \mathrm{mg}$ of lipid (SPC) was dissolved in $456 \mu \mathrm{l}$ of ethanol. Nimodipine (30 mg) was then added to the lipid solution at $70^{\circ} \mathrm{C}$. Drug lipid solutions then added to the vial which containing cholesterol (30 mg for low lipid and 60 $\mathrm{mg}$ for high lipid formulation), and the temperature was kept at $70^{\circ} \mathrm{C}$ using a water bath. To this drug lipid solution, $1.5 \mathrm{ml}$ of HPLC water was added (primary hydration step) and agitated for 2 minutes 
using a WhirlMixer TM (Fisher Scientific, UK) to achieve concentrated suspension of liposomes. Liposomes were produced by adding the rest of the aqueous phase, which was $11.5 \mathrm{ml}$ of water plus $2 \mathrm{ml}$ of phosphate buffer called secondary hydration step and vortexed for a further 2 minutes. The generated liposomal pellets were kept for 1 hour at $25^{\circ} \mathrm{C}$ to anneal before using for subsequent studies. ${ }^{16}$ Chitosan coated nimodipine-loaded liposome formulation was prepared by including chitosan glutamate (PROTASAN ${ }^{\circledR}$ UPG213) to liposomes generated from ethanol-based proliposomes. The mucoadhesive solution was prepared by dissolving $30 \mathrm{mg}$ of chitosan glutamate

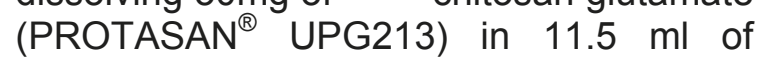
HPLC water. The chitosan solution was used in the secondary step of hydration to generate mucoadhesive liposomes. The formulation was left for 1 hour at $25^{\circ} \mathrm{C}$ to anneal before using. A blank liposome formulation (drug-free liposomes) was also prepared for comparison.

\section{Size reduction of liposomes}

Size reduction of liposomes was performed by probe-sonication (Sonics Vibra-cellCV33, USA). The probe sonicator was placed in the center of the beaker containing $30 \mathrm{~mL}$ of liposomal dispersion. The beaker containing liposomal dispersion was kept in an ice bath to prevent heat damage, which is generated during size reduction. The reduction size of particles run at $30 \%$ amplitude for 10 minutes. ${ }^{17}$

\section{Determination of particle size}

The particle size distribution of liposomes was measured using laser diffraction
(Malvern Mastersizer 2000, Malvern Instruments Ltd., UK) and also photon correlation spectroscopy (Zetasizernano, Malvern Instruments Ltd., UK). The average value of the pellet size was expressed as the mean volume diameter (D0.5). The polydispersity was expressed as a Span factor, and a high Span value indicates a wide size distribution of particles.

Span $=($ D0.9-D0.1)/D0.5 ............. Eq. 1)

Where D0.9; 90\% undersized, D0.5; 50\% undersized, and D0.1; $10 \%$ undersized. ${ }^{18}$

\section{Determination of zeta potential}

The zeta potential of the prepared formulations was measured using a zetasizernano instrument (Malvern Instruments Ltd., UK). The phosphate buffer solution was used to hydrate proliposomes and then appropriately diluted with distilled water. A zetasizer cell filled by $0.8 \mathrm{ml}$ of the liposomal dispersion and surface charge of the pellets was measured using Malvernzetasizer. The results are expressed as mean \pm standard deviation for at least three different batches of each liposome formulation. ${ }^{19}$

\section{Determination of drug entrapment efficiency}

The entrapment efficiency was measured by passing $1 \mathrm{ml}$ of the liposome dispersion through syringe filters $(0.22 \mu \mathrm{m})$ using a 5 $\mathrm{ml}$ syringe. Then, the filter was washed out by passing $2 \mathrm{ml}$ of water. To this aqueous dispersion of passed liposomes, $7 \mathrm{ml}$ of methanol was added, followed by shaking. The obtained solution was quantified by HPLC and considered as the entrapped

Table 1: Liposome formulations.

\begin{tabular}{lccccc}
\hline Formula & $\begin{array}{c}\text { Lipid SPC } \\
(\mathbf{m g})\end{array}$ & $\begin{array}{c}\text { Cholesterol } \\
(\mathbf{m g})\end{array}$ & $\begin{array}{c}\text { Ethanol } \\
(\boldsymbol{\mu l})\end{array}$ & $\begin{array}{c}\text { Nimodipine } \\
(\mathbf{m g})\end{array}$ & $\begin{array}{c}\text { Chitosan } \\
(\mathbf{m g})\end{array}$ \\
\hline Low lipid formulation & 120 & 30 & 228 & 30 & - \\
High lipid formulation & 240 & 60 & 456 & 30 & - \\
$\begin{array}{l}\text { Chitosan coated Nimodipine- } \\
\text { loaded liposomes }\end{array}$ & 240 & 60 & 456 & 30 & 30 \\
\begin{tabular}{l} 
Drug-free liposomes \\
\hline
\end{tabular} & 240 & 60 & 456 & - & - \\
\hline
\end{tabular}


fraction. Simultaneously, the filter was dipped in $4 \mathrm{ml}$ of methanol, and then the fraction which did not pass through the filter was pushed and pulled out through syringe. The resultant of the methanolic solution was then similarly analyzed by HPLC (Agilent 1200, USA) and was regarded as un-entrapped fraction. ${ }^{20}$ The entrapment efficiency (EE) was finally calculated as:

$$
\mathrm{EE} \mathrm{\%}=\frac{\text { Entrapped fraction }}{\substack{\text { Entrapped fraction }+ \\ \text { Un-entrapped fraction }}}
$$

\section{Permeability measurement}

Permeability of the liposome dispersion was measured using ultra-thin silicone membrane (J-flex Rubber Products, UK) which was pre-dipped in phosphate buffer saline ( $\mathrm{pH}$ 6.5). Two Franz cells were taken and filled with $3.5 \mathrm{ml}$ of phosphate buffer saline ( $\mathrm{pH}$ 6.5). A small magnetic stirrer was placed at the bottom of the Franz cell. Ultra-thin silicone membrane was placed between the donor (cap), and acceptor of the Franz cell then fixed with a clip. These cells were then placed on a magnetic plate water bath (JB Aqua 26 Plus, Grant Instruments, UK) at $37^{\circ} \mathrm{C} \pm 1$ and motor was allowed to rotate the magnetic bar at $600 \mathrm{rpm}$. One $\mathrm{ml}$ of sonicated nimodipine-loaded nanoliposome (without chitosan) added to one Franz cell, and one $\mathrm{ml}$ of sonicated chitosan coated nimodipine -loaded nanoliposome dispersion added to the other Franz cell.0.2 $\mathrm{ml}$ of the samples from each cell were collected at time intervals of $1,2,5,10,30,60,90,120$, 150, 180, 210 and 240 min using HPLC syringe and placed them into HPLC vials. ${ }^{21}$ To each of the samples, $0.8 \mathrm{ml}$ of methanol was added and quantified using HPLC at a wavelength $237 \mathrm{~nm}^{22}$

\section{Statistical analysis}

Statistical analysis was performed using the statistical package for the social sciences statistics software (version 21). All experiments were carried out in triplicate, and the results were reported as mean $\pm S D$. Analysis of variance (ANOVA) and Student's t-tests (paired t-tests) were used to determine the difference between samples. The variation was considered statistically significant when the obtained $P$ value was less than 0.05 .

\section{Results}

Particle size and size distribution of nimodipine loaded liposomes

The effect of lipid concentrations, loading drug and inclusion of mucoadhesive agents into liposome formulation on the size and size distribution of liposomes generated from soya phosphatidylcholine and cholesterol were measured both before and after probe sonication as shown in Table 2. Probe sonication decreased the size and size distribution of pellets significantly $(P<0.05)$, since the size of liposomes reduced from micro size range to the nano size range except for size distribution of liposome free drug formulation, was not changed significantly $(P=0.592)$ (Table 2).

\section{Zeta potential}

Table 3 demonstrated the zeta potential of liposome formulations was measured for all the formulations before and after probe sonication. The zeta potential for drug loaded liposome in both low and high lipid concentrations was in negative level, and there were not significantly different. Whilstin chitosan coated liposome formulation, the zeta potential was positive $(13.803 \pm 0.195 \mathrm{mV})$ and significantly increased in the surface charge of pellets compared to the non-coated chitosan formulation of high lipid content $(P=0.001)$ as shown in Table 3.

\section{Entrapment efficiency of nimodipine}

The drug entrapment efficiency of nimodipine loaded in liposomes before and after probe sonication was measured using high performance liquid chromatography (HPLC) following the separation of the nonentrapped drug from liposome entrapped fraction of the drug (Table 4). The loading drug efficiency was between $63.313 \pm$ $1.077 \%$ to $76.033 \pm 0.094 \%$ for nimodipine 
loaded liposomes with low lipid content, and chitosan coated nimodipine-loaded liposomes respectively. Inclusion of chitosan to the liposome formulation significantly increased in loading drug efficiency $(P=0.001)$. The loading drug efficiency reduced significantly after probe sonication $(P<0.05)$ (Table 4$)$.

Table 2: Particle size and size distribution of the liposome formulations.

\begin{tabular}{lccc}
\hline Liposome formulations & $\begin{array}{c}\text { Entrapment efficiency } \\
\text { Before probe sonication } \\
(\%)\end{array}$ & $\begin{array}{c}\text { Entrapment efficiency } \\
\text { after probe sonication } \\
(\%)\end{array}$ & $P$ value \\
\hline $\begin{array}{l}\text { Nimodipine-loaded liposomes } \\
\text { with low lipid content }\end{array}$ & $63.313 \pm 1.077$ & $52.423 \pm 1.195$ & $<0.001$ \\
$\begin{array}{l}\text { Nimodipine-loaded liposomes } \\
\text { with high lipid content }\end{array}$ & $66.957 \pm 3.641$ & $57.461 \pm 1.287$ & 0.013 \\
$\begin{array}{l}\text { Chitosan coated Nimodipine- } \\
\text { loaded liposomes }\end{array}$ & $76.033 \pm 0.094$ & $69.762 \pm 0.216$ & $<0.001$ \\
\hline
\end{tabular}

Table 3: Zeta potential of the liposome formulations.

\begin{tabular}{lccc}
\hline Liposome formulation & $\begin{array}{c}\text { Zeta Potential }(\mathbf{m V}) \\
\text { Before probe-sonication } \\
\text { Mean SD }\end{array}$ & $\begin{array}{c}\text { After probe-sonication } \\
\text { Mean + SD }\end{array}$ & $\boldsymbol{P \text { value }}$ \\
\hline Drug free liposomes & $-4.21 \pm 1.256$ & $-1.655 \pm 0.629$ & 0.401 \\
$\begin{array}{l}\text { Nimodipine-loaded liposomes } \\
\text { with low lipid content }\end{array}$ & $-3.21 \pm 1.003$ & $-2.02 \pm 0.649$ & 0.160 \\
$\begin{array}{l}\text { Nimodipine-loaded liposomes } \\
\text { with high lipid content }\end{array}$ & $-1.960 \pm 1.446$ & $-1.317 \pm 0.153$ & 0.486 \\
$\begin{array}{l}\text { Chitosan coated Nimodipine- } \\
\text { loaded liposomes }\end{array}$ & $13.803 \pm 0.195$ & $5.286 \pm 0.341$ & $<0.001$ \\
\hline
\end{tabular}

Table 4: Entrapment efficiency of drug loaded liposome formulations.

\begin{tabular}{|c|c|c|c|c|c|c|}
\hline \multirow[b]{2}{*}{$\begin{array}{l}\text { Liposome } \\
\text { formulation }\end{array}$} & \multicolumn{2}{|c|}{ Volume mean diameter (VMD) } & \multicolumn{3}{|c|}{ Polydispersity of particles } & \multirow[b]{2}{*}{$\begin{array}{c}P \\
\text { value }\end{array}$} \\
\hline & $\begin{array}{l}\text { Before probe- } \\
\text { sonication } \\
(\mu \mathrm{m})\end{array}$ & $\begin{array}{l}\text { After probe- } \\
\text { sonication } \\
(\mathrm{nm})\end{array}$ & $\begin{array}{c}P \\
\text { value }\end{array}$ & $\begin{array}{l}\text { Before probe- } \\
\text { sonication } \\
\text { (Span) }\end{array}$ & $\begin{array}{l}\text { After probe- } \\
\text { sonication } \\
\text { (PDI) }\end{array}$ & \\
\hline $\begin{array}{l}\text { Drug free } \\
\text { liposomes }\end{array}$ & $2.5450 \pm 0.056$ & $122.40 \pm 0.015$ & $<0.001$ & $1.7040 \pm 0.042$ & $2.0000 \pm 0.070$ & 0.592 \\
\hline $\begin{array}{l}\text { Nimodipine-loaded } \\
\text { liposomes with low } \\
\text { lipid content }\end{array}$ & $6.3070 \pm 0.123$ & $119.41 \pm 0.007$ & $<0.001$ & $6.2066 \pm 1.071$ & $0.4223 \pm 0.066$ & 0.008 \\
\hline $\begin{array}{l}\text { Nimodipine-loaded } \\
\text { liposomes with } \\
\text { higher lipid content }\end{array}$ & $3.6066 \pm 0.513$ & $114.09 \pm 0.025$ & $<0.001$ & $7.5960 \pm 2.086$ & $0.3387 \pm 0.075$ & 0.003 \\
\hline $\begin{array}{l}\text { Chitosan coated } \\
\text { Nimodipine-loaded } \\
\text { liposomes }\end{array}$ & $2.9290 \pm 0.076$ & $122.48 \pm 0.002$ & $<0.001$ & $5.9706 \pm 0.111$ & $0.4586 \pm 0.026$ & $<0.001$ \\
\hline
\end{tabular}




\section{Permeability studies of nimodipine}

The permeability study in the present research was performed using ultra-thin silicone membrane and was determined by obtaining the drug release profile of both drug loaded liposome and chitosan coated drug-loaded liposome formulation, as shown in Figure 1. The permeability of the drug through the membrane improved with chitosan coated liposome formulations, and drug permeability significantly increased at $240 \mathrm{~min}$ compared to non-coated formulation $(P=0.0361)$.

\section{Discussion}

\section{Particle size and size distribution of} liposomes

The particle size of the liposomes has been considered an important factor for delivery of drugs. The particle sizes of liposome formulations and size distribution before and after probe sonication are shown in Table 2. Probe sonication resulted in a significant decrease in particle size $(P<0.05)$ for all the liposome formulations (Table 2) and varied in the range of $114 \mathrm{~nm}$ $-122.4 \mathrm{~nm}$. The volume mean diameter (VMD) of nimodipine-loaded liposomes with low lipid content (119.41 $\mathrm{nm} \pm 0.007)$ and with higher lipid content (114.09 $\mathrm{nm} \pm$ $0.025)$ were almost in similar range $(P=0.3577)$, however these were found to slightly lower than the VMD of drug free liposomes (122.40 $\mathrm{nm} \pm$ 0.015). On the other hand, the formulation with chitosan coating exhibited VMD of (122.48 $\mu \mathrm{m} \pm$ 0.002) which was higher than the VMD of formulations with varying lipid concentrations $(P<0.05)$. However, it was found to be in equal range compare to blank liposomes $(P>0.05)$. This suggests that chitosan coating increased the size of liposomes marginally and it is supported by studies performed by Guo co-workers, 2003, who studied the effect of chitosan concentration and lipid type on the chitosan coated liposome. . $^{24,24}$ Polydispersity of drug loaded liposome pellets (low lipid, high lipid, and chitosan coated liposome formulation) before probe sonication was significant $(P<0.05)$ compare to the drug free liposome formulation. In similarity to particle size, probe-sonication resulted in higher PDI for the chitosan coated nimodipineloaded liposomes (0.4586 \pm 0.026$)$ in comparison to the other drug loaded liposome formulations (Table 2). However, the difference was not found to be

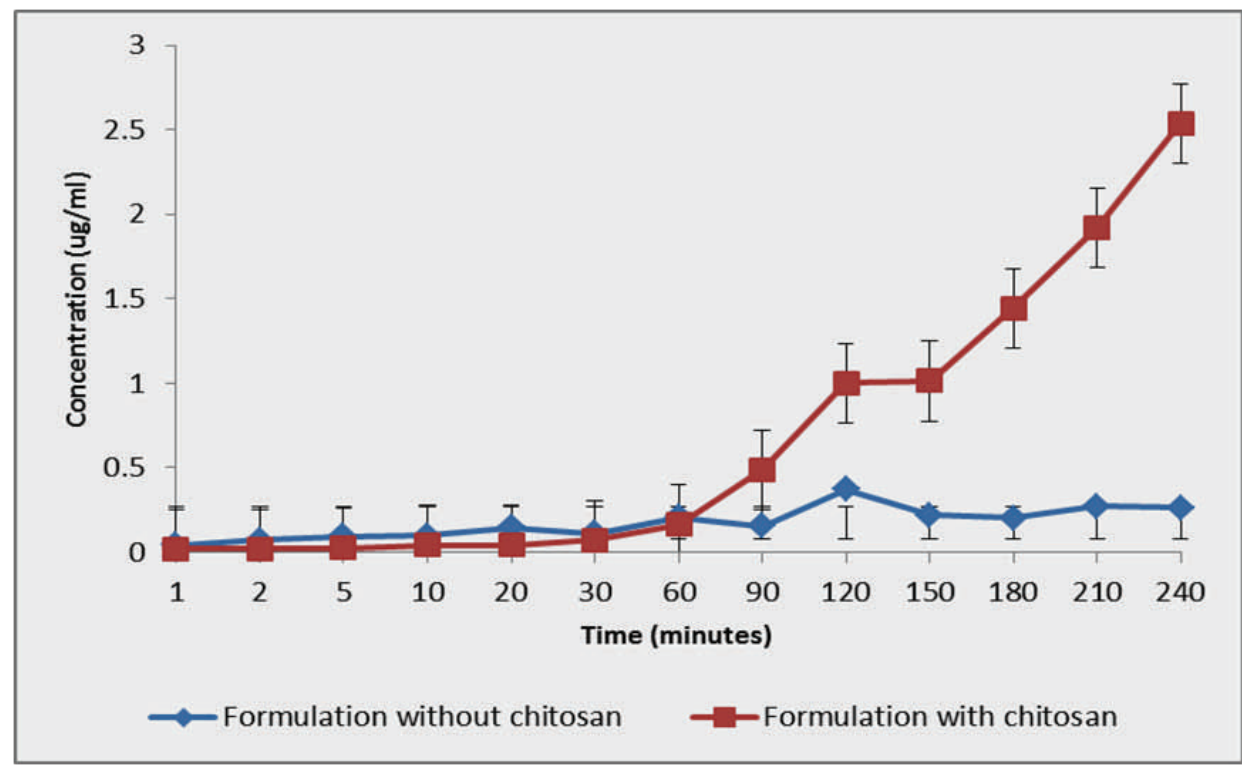

Figure 1: In vitro penetration-time profile of chitosan coated and non-chitosan coated liposome formulation $(P=0.0361$ at $240 \mathrm{~min})$ 
statistically significant $(P=0.9412)$. In accordance with the preliminary literature findings, this difference in PDI might be attributed to the increased concentration of the cholesterol in the formulation which would have enhanced stability to the bilayers in comparison to other formulations thereby avoiding disruption of the vesicles. ${ }^{25,26}$

\section{Zeta potential}

Zeta potential is the difference between the dispersion medium and the stationary layer of fluid attached to the dispersed particle. It provides an indication related to the potential stability of the respective colloidal system. ${ }^{24}$ Zeta potential values of more than $+30 \mathrm{mV}$ or lower than- $30 \mathrm{mV}$ suggest stronger repulsion between the particles and thus fewer tendencies for the particles to adhere together. ${ }^{27}$ The zeta potential of all the formulations except the chitosan coated formulation was found to be in the slight negative range (Table 3 ) both before and after probe-sonication. The zeta potential of liposomes coated by chitosan changed significantly $(P=0.001)$ from slightly negative to positive values (13.803 $\mathrm{mV} \pm 0.195)$ by the inclusion of chitosan into liposome formulation. The positively charged surface of chitosan coated liposomesis contributed to the highly positive charge carried by chitosan, which favors adhesion to the cells membranes. $^{24,28}$ Furthermore, the zeta potential was found to be reduced (less negative value) on increasing the concentration of the lipid. However, there was no significant difference observed among both formulations with varying lipid concentrations $(P=0.286)$. This finding is in agreement with the studies carried out by Szczes, $2013 .{ }^{29}$ Overall, the zeta potential was not changed significantly among the formulations with varying lipid concentrations $(P>0.05)$. The differences in zeta potential among all the liposome formulations before probe-sonication compared to after probe-sonication were found to be statistically significant $(P<0.05)$.

\section{Entrapment efficiency}

Nimodipine is a hydrophobic drug, and it is distributed in the phospholipid bilayers rather than the aqueous core of liposomes. Table 4 clearly shows the chitosan coated formulation with maximum nimodipine entrapment $(76.033 \% \pm 0.094)$, which was found to be significantly higher among all three formulations $(P<0.05)$. The result in accordance with a study conducted by Singla et al., 2001, who demonstrated increased entrapment efficiency of nifedipine microspheres when coated with chitosan. ${ }^{30}$ Increased drug entrapment can be explained on the basis that incorporation of chitosan into formulation enhances its viscosity, which further prevents the escape of the drug molecules from the droplets. ${ }^{31}$ Lowest entrapment efficiency was given by the formulation with lower lipid content $(63.313 \% \pm 1.077)$, and the loading efficiency of nimodipine increased when the lipid concentration increased. The obtained result was in agreement with studies conducted by Wang, et al. 2006, who explored increased drug entrapment with increasing lipid concentration to the formulation. ${ }^{11}$ Probe sonication caused significantly $(P<0.05)$ decreased drug entrapment in all formulations (Table 4). The decreasing of drug encapsulation may be caused by possible degradation of phospholipids and leakage of the drug during size reduction. ${ }^{32}$

\section{Permeability study}

The permeability of chitosan coated nimodipine-loaded liposomes was much higher than the liposomes without chitosan, as shown in Figure 1. This difference between the two formulations was found to be statistically significant $(P<0.05)$ after $240 \min (P=0.0361)$. Chitosan has been considered as permeability enhancing agenton the basis of ability to open tight junctions of the membrane..$^{33,34}$ The opening of tight junctions was allowed due to the interaction of negatively charged sites of cell membranes with the positively charged amino group attached on the 
C-2 position of chitosan. ${ }^{34}$ The maximum drug permeation through the membrane shown by the chitosan coated nimodipineloaded liposomes was $2.53 \mu \mathrm{g} / \mathrm{ml}$ in the duration of four hours. The permeation of the drug molecules for the formulation without coating through the silicone membrane was almost similar throughout four hours' time-period.

\section{Conclusion}

Liposomes have been suggested as one of the novel and efficient drug delivery systems through various research studies. This study also contributed to it by preparing and characterizing nimodipineloaded liposomes. The present study reflected the suitability and efficiency of ethanol based proliposome method to prepare the nimodipine-loaded liposomes. High lipid contained formulation provided maximum entrapment efficiency of the drug. Reduction of particle size using probe sonication showed a dramatic effect on the drug loading efficiency. This study explored that liposomes with higher lipid concentration and chitosan coating can be considered as efficient carriers for the nose to brain delivery of nimodipine. Additionally, chitosan can be regarded as drug entrapment and permeability enhancer.

\section{Competing interests}

The authors declare that they have no competing interests.

\section{References}

1. Choudhary R, Goswami L. Nasal route: A novelistic approach for targeted drug delivery to CNS. Int Res J Pharm 2013; 4:59-62.

2. Illum L. Nasal drug delivery: new developments and strategies. Drug Discov Today 2002; 7(23):1184-9.

3. Upadhyay P, Trivedi J, Pundarikakshudu K, Sheth N. Direct and enhanced delivery of nanoliposomes of anti schizophrenic agent to the brain through nasal route. Saudi Pharm J SPJ 2017; 25(3):346-58.

4. Chen KH, Di Sabatino M, Albertini B, Passerini N, Kett VL. The effect of polymer coatings on physicochemical properties of spray-dried liposomes for nasal delivery of BSA. Eur J Pharm Sci Off J Eur Fed Pharm Sci 2013; 50(3-4):31222.
5. Khan AR, Liu M, Khan MW, Zhai G. Progress in brain targeting drug delivery system by nasal route. J Control Release Off $\mathrm{J}$ Control Release Soc 2017; 268:364-89.

6. Patil SB, Sawant KK. Development, optimization and in vitro evaluation of alginate mucoadhesive microspheres of carvedilol for nasal delivery. J Microencapsul 2009; 26(5):432-43.

7. Ugwoke MI, Agu RU, Verbeke N, Kinget R. Nasal mucoadhesive drug delivery: background, applications, trends and future perspectives. Adv Drug Deliv Rev 2005;57(11):1640-65.

8. Amin M, Jaafari MR, Tafaghodi M. Impact of chitosan coating of anionic liposomes on clearance rate, mucosal and systemic immune responses following nasal administration in rabbits. Colloids Surf B Biointerfaces 2009; 74(1):225-9.

9. Bonferoni MC, Giunchedi P, Scalia S, Rossi S, Sandri G, Caramella C. Chitosan gels for the vaginal delivery of lactic acid: Relevance of formulation parameters to mucoadhesion and release mechanisms. AAPS Pharm Sci Tech 2006; 7(4):E141-7.

10. Luppi B, Bigucci F, Cerchiara T, Zecchi V. Chitosan-based hydrogels for nasal drug delivery: from inserts to nanoparticles. Expert Opin Drug Deliv 2010; 7(7):811-28.

11. Wang $Z$, Deng $Y$, Zhang $X$, Wang $T$, Wu F. Development and pharmacokinetics of nimodipine-loaded liposomes. J Pharm Pharmacol 2006; 58(9):1289-94.

12. Song $X$, Jiang $Y$, Ren $C$, Sun $X$, Zhang $Q$, Gong T, et al. Nimodipine-loaded mixed micelles: formulation, compatibility, pharmacokinetics, and vascular irritability study. Int $\mathrm{J}$ Nanomedicine 2012; 7:3689-99.

13. Tomassoni D, Lanari A, Silvestrelli G, Traini E, Amenta F. Nimodipine and its use in cerebrovascular disease: evidence from recent preclinical and controlled clinical studies. Clin Exp Hypertens 2008; 30(8):744-66.

14. Soliman GM, Sharma R, Choi AO, Varshney SK, Winnik FM, Kakkar AK, et al. Tailoring the efficacy of nimodipine drug delivery using nanocarriers based on A2B miktoarm star polymers. Biomaterials 2010; 31(32):8382-92.

15. Yang D, Zhu J, Zheng Y, Ge L. Preparation, Characterization, and Pharmacokinetics of Sterically Stabilized Nimodipine-Containing Liposomes. Drug Dev Ind Pharm 2006; 32(2):219 $-27$.

16. Elhissi AMA, Karnam KK, Danesh-Azari M-R, Gill HS, Taylor KMG. Formulations generated from ethanol-based proliposomes for delivery via medical nebulizers. J Pharm Pharmacol 2006; 58(7):887-94.

17. Schroeder A, Kost J, Barenholz Y. Ultrasound, liposomes, and drug delivery: principles for using ultrasound to control the release of drugs from liposomes. Chem Phys Lipids 2009; 162(1):1-16. 
18. Gavini E, Hegge AB, Rassu G, Sanna V, Testa C, Pirisino G, et al. Nasal administration of carbamazepine using chitosan microspheres: in vitro/in vivo studies. Int J Pharm 2006; 307(1):9 $-15$.

19. Jaafar-Maalej C, Diab R, Andrieu V, Elaissari A, Fessi $H$. Ethanol injection method for hydrophilic and lipophilic drug-loaded liposome preparation. J Liposome Res 2010; 20(3):228-43.

20. Nii T, Ishii F. Encapsulation efficiency of water-soluble and insoluble drugs in liposomes prepared by the microencapsulation vesicle method. Int J Pharm 2005; 298(1):198-205.

21. Zhang H, Zhu X, Shen J, Xu H, Ma M, Gu W, et al. Characterization of a liposome-based artificial skin membrane for in vitro permeation studies using Franz diffusion cell device. J Liposome Res 2016; 27:1-34.

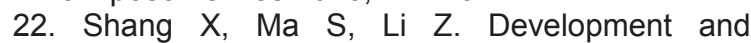
Validation of a RP-HPLC method for determination of nimodipine in sustained release tablets. Journal of Chemistry 2013; 2013:612082.

23. Guo J, Ping Q, Jiang G, Huang L, Tong $Y$. Chitosan-coated liposomes: characterization and interaction with leuprolide. Int J Pharm 2003; 260(2):167-73.

24. Mady MM, Darwish MM. Effect of chitosan coating on the characteristics of DPPC liposomes. J Adv Res 2010; 1(3):187-91.

25. Liang X, Mao G, Ng KYS. Mechanical properties and stability measurement of cholesterolcontaining liposome on mica by atomic force microscopy. J Colloid Interface Sci 2004; 278(1):53-62.

26. Nie Y, Ji L, Ding H, Xie L, Li L, He B, et al. Cholesterol derivatives based charged liposomes for doxorubicin delivery: preparation, in vitro and in vivo characterization. Theranostics 2012; 2(11):1092-103.

27. Paolino D, Sinha $P$, Fresta M, Mauro F. Drug Delivery Systems - Encyclopedia of Medical Devices and Instrumentation - Paolino - Wiley Online Library; 2006.

28. Khatri K, Goyal AK, Gupta PN, Mishra N, Mehta A, Vyas SP. Surface modified liposomes for nasal delivery of DNA vaccine. Vaccine 2008; 26(18):2225-33.

29. Szcześ A. Effects of DPPC/Cholesterol liposomes on the properties of freshly precipitated calcium carbonate. Colloids Surf B Biointerfaces 2013; 101:44-8.

30. Singla AK, Sharma ML, Dhawan S. Nifedipine loaded chitosan microspheres: characterization of internal structure. Biotech Histochem Off Publ Biol Stain Comm 2001; 76(4):165-71.

31. Sinha V, Singla A, Wadhawan S, Kaushik R, Kumria R, Bansal K, et al. Chitosan microspheres as a potential carrier for drugs. Int J Pharm 2004; 274(1-2):1-33.

32. Akbarzadeh A, Rezaei-Sadabady R, Davaran S, Joo SW, Zarghami N, Hanifehpour $\mathrm{Y}$, et al.
Liposome: classification, preparation, and applications. Nanoscale Res Lett 2013; 8(1):102.

33. Ahmed TA, Aljaeid BM. Preparation, characterization, and potential application of chitosan, chitosan derivatives, and chitosan metal nanoparticles in pharmaceutical drug delivery. Drug Des Devel Ther 2016; 10:483507.

34. Sonaje K, Chuang E-Y, Lin K-J, Yen T-C, Su F-Y, Tseng MT, et al. Opening of epithelial tight junctions and enhancement of paracellular permeation by chitosan: microscopic, ultrastructural, and computed-tomographic observations. Mol Pharm 2012; 9(5):1271-9. 